\title{
Parental duties and untreatable genetic conditions
}

Henriikka Clarkeburn Glasgow University, Glasgow

\begin{abstract}
This paper considers parental duties of beneficence and non-maleficence to use prenatal genetic testing for non-treatable conditions. It is proposed that this can be a duty only if the testing is essential to protect the interests of the child ie only if there is a risk of the child being born to a life worse than non-existence. It is argued here that non-existence can be rationally preferred to a severely impaired life. Uncontrollable pain and a lack of any opportunity to develop a continuous self are considered to be sufficient criteria for such preference. When parents are at risk of having a child whose life would be worse than non-existence, the parents have a duty to use prenatal testing and a duty to terminate an affected pregnancy. Further, such duty does not apply to any conditions where the resulting life can be considered better than non-existence.

(Fournal of Medical Ethics 2000;26:400-403)
\end{abstract}

Keywords: Prenatal testing; parental duties; beneficence; non-maleficence.

\section{Introduction}

New genetic knowledge increases the possibility of using prenatal testing for a wide range of genetic disorders. These new opportunities may produce great benefits to those parents who wish to avoid having a child with a genetic defect, but they are also a source of concern. Part of this concern is a fear that the introduction of prenatal testing technology will limit reproductive freedom by imposing new parental duties.

The focus of this paper is whether parents who are at risk of having an abnormal child, have a moral duty to acquire genetic information about their fetus. The scope is limited to genetic defects that have no available therapy or cure, and where selective abortion is the only "preventive" method. Also the possible parental duty is considered only in the perspective of parental duties towards their child and not as a possible duty towards society.

The research framework is based on duties of beneficence and non-maleficence. The special interest is whether these duties can be extended to cover acts where the "harm" primarily consists of bringing a child into existence. If this is a sensible extension of the duty of beneficence, the following task is to analyse the boundaries of such duty.

\section{Parental duties}

The principles of beneficence and non-maleficence can be divided into four distinguishable obligations:

- One ought not to inflict harm or evil (nonmaleficence)

- One ought to prevent evil and harm

- One ought to remove evil and harm

- One ought to do or promote good

In relation to reproduction, duties of nonmaleficence include not harming the already existing fetus and not knowingly creating a fetus who will exist in a harmed state. Duties of beneficence require parents to remove or ameliorate existing harm.

To explicate the duty of beneficence or nonmaleficence, harm will have to be understood in the sense of setting back the interests of one party by intentional and unintentional actions on behalf of another party. A necessary element in all harming is that it has an effect on someone's interests, and these interests are distinguishable components of a person's good or wellbeing. Some interests, often called "welfare interests", are more basic than the others in a sense that when they are severely set back, no other interests in a person's interestnetwork can advance. A severe genetic abnormality can be considered to set back welfare interests. A more moderate abnormality can set back this or that particular interest of a person, without necessarily setting back basic welfare interests. Acts of beneficence in relation to prenatal testing, deal with the adverse influences to the welfare interests of the future child.

For genetic testing to become a parental duty, it is required that the interests of the offspring would be best served, and that offspring would be better off, if the parents did participate in genetic testing. To justify a duty of beneficence to use prenatal genetic testing also requires that the best interests of the child cannot be promoted in any other way and that it is essential to the protection of the child's interests.

A duty to acquire information about the genetic constitution of the fetus must be connected to a duty to act upon that information. It would be illogical to suggest that there is a duty to use prenatal testing to confirm the genetic constitution of a fetus, if there was no duty to use this information to benefit the child. In the case of non-preventable and untreatable genetic defects, the only possible 
benefiting action, from the point of view of the fetus, is a termination of an affected pregnancy. Justification is needed to prove that termination of a pregnancy can be in the best interests of the child who would otherwise develop.

It is not obvious, however, that termination of a pregnancy can be a beneficial act from the point of view of the future child. This type of "prevention" is problematic in at least two different ways: first we have to understand how procreation can be a harmful act and second, if there are ever conditions, when non-existence can rationally be preferred to life.

\section{Harming and procreation}

In the case of incurable and untreatable genetic abnormalities, the options for existence are either 1) existence with the genetic abnormality, or 2) non-existence. The only alternative to genetically abnormal existence is a termination of an affected pregnancy, after which the "original" child who would have been born with the genetic abnormality, would not be born at all. In the case of untreatable genetic conditions, the abnormal and potentially "harming" genes are an inseparable and essential part of the affected fetus. There is no option for existing without that complete genetic constitution.

For giving birth to be a harmful act, it must meet the counterfactual condition of harming. ${ }^{2}$ In other words, giving birth to a child can be considered an act of harming if and only if doing $\mathrm{X}$ (conceiving and carrying to term a child with the impairment) causes B's (the resulting child) personal interests to be in a worse condition than if not doing $\mathrm{X}$. If the child's life is better than non-existence, then parents doing $\mathrm{X}$ have not caused the child to be worse off than not doing $\mathrm{X}$, and therefore the parents have not harmed their child by bringing it into existence.

If, on the other hand, being born is worse than non-existence, the parental act of carrying to term a child with such a severe impairment can be considered as a harmful act. The parental duty of beneficence to use prenatal testing in conjunction with a possible termination of an affected pregnancy, can therefore only be sustained if it can be proved that it is possible 1) to have a life worse than non-existence, and 2) to detect an affected genetic constitution before conception or birth. If we can prove that a condition exists that is worse than non-existence, and if the use of prenatal diagnosis and acting according to that knowledge is the only way to protect the child's best interests, then parents' duty of beneficence towards their future child requires them to know this particular genetic information. If being born inflicts intolerable harm on the child, and there is a form of prevention in relation to financial and technical abilities and opportunities, the parental duty of beneficence requires such preventive measures to be taken.
Can life be worse than non-existence?

Stating that life can be worse than non-existence refers to an assumption that living a life with certain impairments is worse than never coming into existence at all. Such intolerable life is defined by a negative balance between the possible benefits and burdens of that life. In intolerable life, pain and physical/intellectual inabilities would outweigh any possible benefits life could offer.

According to some doctrines, all life is of equal value. This incorporates a conviction that life is always better than non-existence, and that a balancing of life's benefits and harms should not be attempted. Doctrines of equal value for all life can be based on either religious criteria of a living soul, or on secular grounds by reference to distinctively human inheritance which is a sufficient criterion for a life worth living. What is common to both of these views is that life is not valuable because of the qualities it may possess, but that it is categorically valuable in itself. Thus even those who are unable to have any mental life or appreciation of joy or comfort have a life worth living. ${ }^{3}$ Using this reasoning, there could be no parental duty of beneficence to avoid bringing a severely handicapped child into existence.

The categorical appreciation of all life as being worth living is based on irrefutable value premises, which I do not wish to attack or support in this paper. Rather, I wish to investigate the possibilities of there being life worse than existence, from the perspective that life as such is not always worth living, and that evaluations of pain and mental/ intellectual capacities are significant in decisions regarding parental duties of beneficence towards their children in cases of untreatable genetic conditions. If we accept the possibility that life can logically be worse than non-existence, by rejecting the a priori value of life, then it is necessary to establish criteria for differentiating life that is worth living from life that is worse than non-existence.

\section{Criteria for life worse than non-existence}

Could a lack of continuing self be a sufficient criterion for life worse than non-existence? To be a continuing self, one must have more than momentary desires or interests. Instead, one must, at some time, be able to see oneself existing over time. ${ }^{4}$ This criterion includes other proposed criteria for life worth living, such as a necessity to have self awareness, self control, and a capacity to relate to others. Being a continuing self would create an indisputable criterion for having a moral standing and a life worth living. But it does not provide a sufficient criterion for life worse than non-existence.

A life without a sense of continuing self may not be particularly exciting or intellectually stimulating, but neither, all this being equal, is it necessarily a life worse than non-existence. The person lacking a sense of continuing self may be indifferent towards the continuation of that life, but it can be a life of sporadic pleasures. The ability to experience momentary pleasure would make such a life better than non-existence. 
Introduction of continuous and non-palliative pain influences the balance dramatically. A life ridden with severe pain, in particular if that is in addition to life of extremely severe mental retardation, is intuitively closer to a life not worth living, a life which there might a duty to prevent from coming into existence, since the avoidance of inflicting pain, when possible, is a prima facie duty of beneficence.

Pain is a fundamentally subjective experience that is not easily open to external observation. We have only a few unobtrusive methods to quantify pain; increase in heart rate, stroke volume and blood pressure and behavioural signs of moaning body tension etc. But these methods can give a high level of confidence in appreciating pain. Also, there is no reason to assume that the experience of pain would be any less for the mentally retarded. ${ }^{5}$

Therefore I propose that the combination of continuous and non-palliative pain and lack of opportunities to develop a continuous self constitutes life worse than non-existence. A parental duty of beneficence can therefore contain a duty to prevent such a life from coming into existence.

\section{Rational preference for non-existence}

It is problematic to decide when life is worse than non-existence. It seems probable that in most cases life, even with suffering, can be preferred to non-existence. Most competent persons, regardless of their physical impairments, do not express regret for having been born (even though they might have a rational preference for having been born without the impairment). The problem is that most of those whose life most often is considered not worth living, are incompetent to make such a judgment themselves. Still this does not make it an irrational preference.

Stating a preference for non-existence over severely impaired existence is exercising a judgment that whatever interests the impaired parties might have, or come to have, would be already doomed to defeat by their present incurable condition. Thus it would be irrational for anyone protecting those interests to prefer continuance of that condition to non-existence. Implicitly this would mean that lateonset disease could not result in life worse than non-existence, because many life interests have had an opportunity to be fulfilled before the dramatic onset of the disease. The interests for non-existence must be safeguarded even when the person whose interests they are, is unable to make the judgment.

\section{Acting on parental duties}

While the logical possibility exists for life worse than non-existence real life examples are very rare. Most commonly tested genetic disorders do not fulfil the criteria for life worse than non-existence. For example, Down syndrome children are not enduring pain and neither do they lack a sense of continuous self, and cystic fibrosis patients' lives are not at risk of being life worse than non-existence as the patients have full command of their intellectual abilities and their pain is not necessarily continuous or non-palliative.

A possible candidate condition to inflict such severe pain with very limited opportunities for intellectual or physical enjoyment is Tay Sachs disease (TSD). A baby born with TSD appears normal at birth, but will die within a few years as a result of progressive neurological damage. Other examples of possible conditions resulting in life worse than non-existence are: 1) Pompe's disease, where a child suffers severe mental retardation and cardiorespiratory difficulties leading to a premature death within the first year; 2) Trisomy 13, which is associated with severe mental retardation, physical malformations and a life span of only a few months, and 3) Gaucher's disease, where a metabolic dysfunction causes mental disorders, enlarged organs and is fatal before the second birthday. All of these conditions are exteremely rare with frequency of live births between 1:20,000 for trisomy 13 and $1: 360,000$ for TSD for general population.

Even though these all are severe genetic conditions, it is not apparent that a child with them would not be able to experience at the very least sporadic pleasures during the short life and that the pains could not be palliated successfully. And in case of doubt, it would be better to err on the side of caution.

The parental duty which requires parents to prevent a life from coming into existence is a very limited duty of beneficence. The genetic conditions currently included by the UK's National Health Service in standard prenatal screening (Down syndrome and cystic fibrosis) do not fulfil the criteria of harm where the resulting life would be worse than non-existence. It is thus morally unsupported to offer these tests "for the benefit of the future child".

But if a condition which is worse than nonexistence were identified, the parental duty of beneficence, in a moral sense, would extend even to those parents with only a minimal chance of having a child with such a condition. However, for a society to increase prenatal testing procedures in order to detect all extremely rare conditions is unlikely to be an acceptable use of public funds. Thus the moral duty is more prominent when parents are aware of an increased risk of having a child with a genetic condition which would lead to that child having a life worse than non-existence. Parents in such a situation would have a duty to take all possible precautions to prevent a child with the condition being born. These precautions include terminating an affected pregnancy. Parents who know of such risks and are not willing to use prenatal testing with the possible termination of an affected pregnancy, should refrain from conceiving. Society should allow for the realisation of this moral duty, by providing testing opportunities either prior to conception or during pregnancy.

This moral duty of beneficence should not, however, be translated into a legal duty. This conclusion is based on the risk of violating basic human rights regarding privacy and starting a family, and due to 
pragmatic problems relating to enforcing such a legally binding duty. What also follows from this analysis is that there are no moral grounds for enforcing prenatal testing for any conditions that do not fulfil the criteria of life worse than nonexistence, including conditions for which most prenatal testing is performed.

\section{Conclusions}

Parents have a duty of beneficence towards their future children. In relation to prenatal testing for incurable genetic conditions, the duty of beneficence can only be upheld when a child would be born to a life worse than non-existence. Intellectual disabilities alone do not suffice as a criterion of a life worse than non-existence, but the inclusion of pain will provide a logical possibility for such a life. Actual examples of genetic conditions which would have such physical and mental disabilities are very rare, if they exist at all. The logical extension of this limited parental duty of beneficence is that most prenatal testing for incurable genetic conditions cannot be enforced on moral grounds.

\section{Acknowledgement}

My sincere thanks to Professor Robin Downie who supported the original research and commented on an early draft of this paper, and to Dr Roger Downie for correcting the English of the earlier version.

Henriikka Clarkeburn, PhD, MPhil, Mss, is Marie Curie Fellow at the Institute of Biomedical and Life Sciences, University of Glasgow, Glasgow.

\section{References}

1 Beauchamp TL, Childress JF. Principles of biomedical ethics [4thed]. New York, Oxford: Oxford University Press, 1994 2 Feinberg J. Wrongful life and the counterfactual element of harming. Social Philosophy \& Policy 1986;4:145-78.

3 Kuhse H, Singer P. Should the baby live? Oxford: Oxford University Press, 1985.

4 Tooley M. Abortion and infanticide. Oxford: Clarendon Press, 1983.

5 Fordham M, Dunn V. Alongside the person in pain. London: Baillière Tindall, 1994. 